\title{
In vitro properties of antimicrobial bromotyrosine alkaloids
}

\author{
Neora Pick, ${ }^{1}$ Mamta Rawat, ${ }^{1,2}$ Dorit Arad, ${ }^{1}$ Jiong Lan, ${ }^{3}$ Junfa Fan, ${ }^{3}$ \\ Andrew S. Kende ${ }^{3}$ and Yossef Av-Gay ${ }^{1}$ \\ ${ }^{1}$ Division of Infectious Diseases, University of British Columbia, Vancouver, BC V5Z 3J5, \\ Canada \\ ${ }^{2}$ Department of Biology, California State University - Fresno, Fresno, CA 937401, USA \\ ${ }^{3}$ Department of Chemistry, University of Rochester, Rochester, NY 14627-0216, USA
}

Received 6 September 2005

Accepted 11 December 2005

\begin{abstract}
A bromotyrosine alkaloid family of antimicrobial agents was synthesized using the known structure of a natural inhibitor of the mycobacterial mycothiol $S$-conjugate amidase (MCA) as a template. This series of compounds represents a novel class of anti-infective agents against Gram-positive pathogens, including mycobacteria and meticillin- and vancomycin-resistant Staphylococcus aureus. The fact that these compounds are active against mycobacterial strains in which the MCA gene is deleted and against Gram-positive bacteria lacking mycothiol suggests the existence of an alternative target for these compounds. One member of this family, EXEG1706, was identified as the lead compound possessing low MICs $\left(2 \cdot 5-25 \mu \mathrm{g} \mathrm{ml}^{-1}\right)$ for several clinical isolates, whilst having low toxicity for THP-1 monocytes and macrophages.
\end{abstract}

\section{INTRODUCTION}

Mycobacterium tuberculosis, the causative agent of tuberculosis (TB), and Staphylococcus aureus are two of several Gram-positive organisms causing significant morbidity and mortality in humans. M. tuberculosis is the second leading cause of death due to pathogenic agents worldwide (Dye et al., 1999; World Health Organization, 2002). TB has again become a potential public health concern, partly because of its prevalence among the immunocompromised AIDS population and the homeless population, and partly because of an increase in drug-resistant $M$. tuberculosis strains worldwide (Negi et al., 2003). S. aureus synthesizes several virulence factors, such as the capsule and protein $\mathrm{A}$, that allow it to evade host immune defences and survive in hostile environments (for a review, see Lowy, 1998). Due to the large number of virulence factors, $S$. aureus is able to cause a variety of diseases, including major skin, soft tissue, respiratory, bone, joint and endovascular disorders. Drug resistance is also a major cause for concern, as the numbers of meticillin-resistant S. aureus (MRSA) infections in intensive care units steadily increased from 1987 to 1997 (Lowy, 1998), and MRSA resistant to intermediate concentrations of glycopeptides, including vancomycin, have been identified

Abbreviations: BCG, Bacille Calmette-Guérin; FACS, fluorescenceactivated cell sorting; MCA, mycothiol S-conjugate amidase; MRSA, meticillin-resistant Staphylococcus aureus; MSH, mycothiol; MSSA, meticillin-sensitive Staphylococcus aureus; $\mathrm{PI}$, propidium iodide; PMA, phorbol myristate acetate; TB, tuberculosis; VRSA, vancomycin-resistant Staphylococcus aureus; VRSH, vancomycin-resistant Staphylococcus haemolyticus.
(Cassone et al., 2004; Mallaval et al., 2004). Considerable emphasis is now being placed on identifying new drug targets and on developing new antimicrobials to treat infections caused by this pathogen.

One potential drug target in mycobacteria is mycothiol (MSH) and MSH-dependent enzymes such as mycothiol $S$ conjugate amidase (MCA). MSH is the major low molecular mass thiol in $M$. tuberculosis and MCA functions in the MSH-dependent detoxification of oxidizing agents, electrophiles and several antibiotics. MSH binds to an alkylating agent (toxin), forming an $S$-conjugate. MCA then cleaves the MSH-toxin conjugate to release a mercapturic acid, which is excreted from the cell, and 1-D-myo-inositol-2amino-2-deoxy- $\alpha$-D-glucopyranoside, which is recycled into MSH biosynthesis (Newton et al., 2000). MCA represents an important new antimicrobial target, as eukaryotes do not have MSH or MCA and instead rely on glutathionedependent pathways for their detoxification processes. MSH and MCA homologues are commonly found in other genera within the Actinomycetales, including Streptomyces, Nocardia and Corynebacterium. Inhibition of MSH-dependent detoxification at the level of MSH-toxin conjugate binding to the MCA active site may represent a potent way of attacking mycobacterial infections.

Nicholas et al. (2001) screened a variety of marine extracts and identified novel alkaloid compounds with the ability to inhibit the mycobacterial MCA; two of these were novel bromotyrosine alkaloids (Nicholas et al., 2001). The second alkaloid, known as compound 4, served as a template for our 
design and synthesis of a series of compounds to be evaluated for antimicrobial activity (Arad \& Av-Gay, 2003). An independent synthesis and evaluation of bromotyrosine alkaloids was also undertaken by Nicholas et al. (2002) in which the synthetic compounds were evaluated for their ability to inhibit MCA activity, but were not tested against other organisms. As none of the above compounds was tested for its biological activity against microbial pathogens, we tested them against a variety of micro-organisms. Here, we describe the synthesis and evaluation of these novel bromotyrosine alkaloids, some of which demonstrated high antimicrobial activity against a number of Gram-positive organisms. We further characterized EXEG1706, a promising compound showing high activity against $S$. spp. and Mycobacteria spp. and low toxicity to mammalian cells.

\section{METHODS}

Bacterial strains. The strains used in this study are shown in Table 1. Organisms were maintained in stock culture at $-70^{\circ} \mathrm{C}$ and freshly streaked for use before each assay. All organisms were grown in brain heart infusion (BHI) medium [solid medium contained $1.5 \%(\mathrm{w} / \mathrm{v})$ agar; Becton Dickinson] with the exception of Escherichia coli (Luria-Bertani broth), Mycobacterium spp. (Middlebrook 7H9 liquid medium and Middlebrook 7H10 solid medium; Difco Laboratories) and Saccharomyces cerevisiae (yeast nitrogen base without amino acids or ammonium sulphate; Becton Dickinson). Streptococcus pneumoniae strains were grown first on sheep's blood agar (PML Microbiologicals) before transfer to BHI.
Synthesis of bromotyrosine compounds. A series of novel bromotyrosine alkaloids was synthesized (Arad \& Av-Gay, 2003) based on the structure of an alkaloid produced by an Australian nonverongid sponge, Oceanapia sp. (Nicholas et al., 2001). The structures of the five compounds that had the best antimicrobial activity are shown in Fig. 1. Synthesis of three of these compounds has been described previously (Kende et al., 2004; Nicholas et al., 2002).

Fig. 2 summarizes the synthesis of EXEG3022, with a starting substrate of 4-hydroxyphenylpyruvic acid, which resulted in EXEG3022 in four synthetic operations. Pyruvic acid was reacted with $\mathrm{O}$-(tetrahydro- $2 \mathrm{H}-$ pyran-2-yl)hydroxylamine in methanol to give a crude $\mathrm{O}$-(tetrahydro$2 \mathrm{H}$-pyran-2-yl)oxime. This was directly coupled in dichloromethane with tert-butyl- $\mathrm{N}$-(6-aminohexyl)guanidinyl bicarbamate in the presence of 1-hydroxybenzotriazole and 1-(3-dimethylaminopropyl)-3ethylcarbodiimide hydrochloride to produce an $80 \%$ yield of the guanidine amide derivative, labelled compound 2 (Wagner et al., 1998). This intermediate was dissolved in dry tetrahydrofuran and stirred with $\mathrm{N}$-bromosuccinimide to give a $76 \%$ yield of the dibromophenol, labelled compound 3. Mitsunobu reaction of the phenol group of compound 3 with tert-butyl- $N$-(4-hydroxybutyl)guanidinyl bicarbamate, diethyl azodicarboxylate and triphenylphosphine in tetrahydrofuran produced the protected bisguanidine at a $37 \%$ yield after recrystallization (compound 4). Finally, all five protecting groups were cleaved using $30 \%$ trifluoroacetic acid in dichloromethane to give the bis(trifluoroacetate) salt of EXEG3022 at an 70\% yield. EXEG1707, which has a similar structure, was prepared in a parallel manner.

Drug dilution. For use in toxicity assays, unless otherwise specified, compounds were prepared as a $10 \mathrm{mg} \mathrm{ml}^{-1}$ solution in methanol and diluted in RPMI to $1 \mathrm{mg} \mathrm{ml}^{-1}$. Further serial dilutions in RPMI containing $5 \%$ fetal calf serum and $1 \%$ glutamine (modified RPMI) gave the appropriate concentrations for individual assays.

Table 1. List of organisms used in this study

\begin{tabular}{|lll|}
\hline Organism & \multicolumn{1}{c|}{ Strain ${ }^{\star}$} & \multicolumn{1}{c|}{ Source or reference* } \\
\hline Bacillus subtilis & W168 & ATCC \\
Enterococcus faecalis & ATCC 376 & ATCC \\
Enterococcus faecium & 1 CDC 2 & CDC \\
Escherichia coli & JM109 & Yanisch-Perron et al. (1985) \\
Mycobacterium smegmatis & MC -155 & W. R. Jacobs, AECOM \\
Mycobacterium bovis BCG & ATCC 35734 & ATCC \\
Saccharomyces cerevisiae & SD4191 & ATCC \\
Staphylococcus aureus & ATCC 27217 & ATCC \\
VRSA & VGH clinical isolate C4/11 & This study \\
MRSA & VGH clinical isolate B-106BB8 & This study \\
MSSA & VGH clinical isolate B-107BB8 & This study \\
MRSA, quinolone resistant & VGH clinical isolate B-96BC10 & This study \\
MRSA, quinolone sensitive & VGH clinical isolate A-105DD3 & This study \\
MRSA, heteroresistant & VGH clinical isolate A-105DD2 & This study \\
Staphylococcus epidermidis & Laboratory strain & ATCC \\
VRSH & VGH clinical isolate 1BA2 1994 & This study \\
Staphylococcus saprophyticus & & This study \\
Streptococcus pneumoniae & ATCC 49619 & ATCC \\
Streptococcus pneumoniae & FC579 & D. P. Speert, UBC \\
& & \\
\hline
\end{tabular}

*AECOM, Albert Einstein College of Medicine, New York, USA; ATCC, American Type Culture Collection; CDC, Centers for Disease Control and Prevention; VGH, Vancouver General Hospital, 899 West 12 Ave, Vancouver, BC V5Z 1M9, Canada; UBC, University of British Columbia. 
<smiles>NC(=O)NC(N)=NCCCCCCNC(=O)/C(Cc1cc(Br)c(OCCCC(N)(F)F)c(Br)c1)=N/O</smiles>

$N$-[[6-aminoiminomethyl)amino]hexyl]-3-[4-(3-aminopropoxy)-

3,5-dibromophenyl]-2-hydroxyiminopropionamide, bis(trifluoroacetic acid) salt

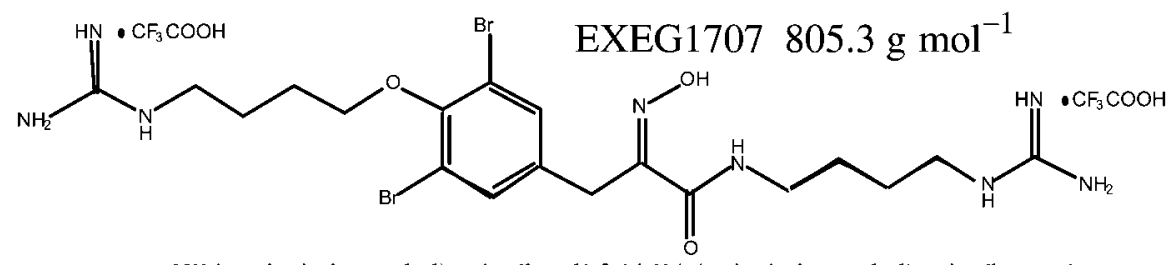

$N$-[4-aminoiminomethyl)amino]butyl]-3-[4-[[4-(aminoiminomethyl)amino]butoxy]-

3,5-dibromophenyl]-2-hydroxyiminopropionamide, bis(trifluoroacetic acid) salt<smiles>NC(=O)O[Mg]O[Mg]</smiles>

$N$-[[6-aminoiminomethyl)amino]hexyl]-3-[4-(3-aminopropoxy)-

3,5-dichlorophenyl]-2-hydroxyiminopropionamide, bis(trifluoroacetic acid) salt

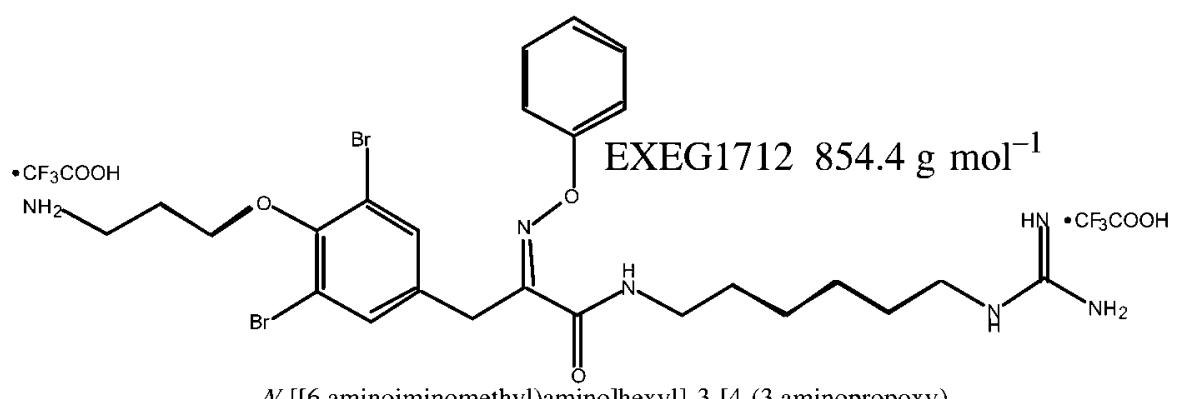

$N$-[[6-aminoiminomethyl)amino]hexyl]-3-[4-(3-aminopropoxy)-

3,5-dibromophenyl]-2-benzyloxyiminopropionamide, bis(trifluoroacetic acid) salt

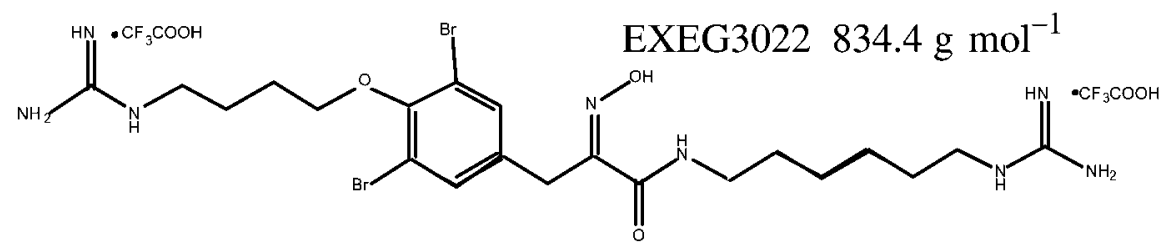

$N$-[[6-aminoiminomethyl)amino] hexyl]-3-[4-[[4-(aminoiminomethyl)amino]butoxy]3,5-dibromophenyl]-2-hydroxyiminopropionamide, bis(trifluoroacetic acid) salt

Fig. 1. Chemical structures and molecular masses of synthetic bromotyrosine alkaloids. Bromotyrosine alkaloids were synthesized as described in Methods. The 5 compounds shown here were the most active out of 30 compounds tested. All molecular masses were calculated for the trifluoroacetic acid salts. 


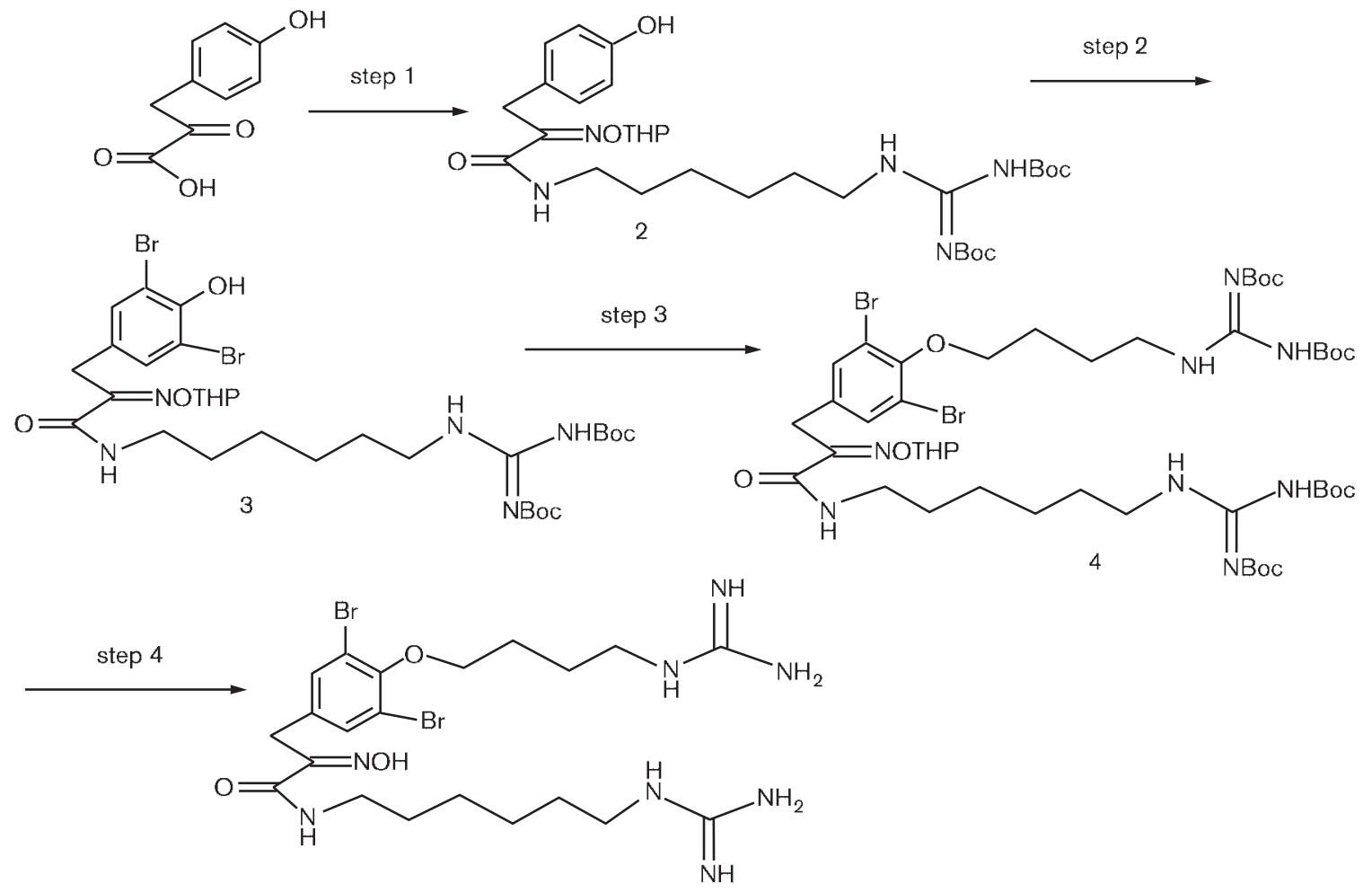

Fig. 2. Description of EXEG3022 synthesis from 4-hydroxyphenylpyruvic acid in four operations; see Methods for details. EXEG1707 was prepared in a parallel manner.

Disk diffusion assay. Test compounds were dissolved at $10 \mu \mathrm{g} \mathrm{ml} \mathrm{l}^{-1}$ in HPLC-grade methanol (Fisher Scientific). Sterile $59 \mathrm{~mm}$ filter paper disks (Difco Laboratories) were impregnated with 5-10 $\mu$ of a compound in solution and allowed to air dry. Test organisms were grown to mid-exponential phase and streaked evenly on to the surface of solid agar using a sterile swab. Disks containing test compounds or solvent alone were applied to the dry agar surface (maximum of six disks per $110 \mathrm{~mm}$ plate) and the plates were incubated at $37^{\circ} \mathrm{C}$ until sufficient bacterial growth had occurred to observe growth inhibition zones, if present (up to 3 days).

For Mycobacterium smegmatis, disk assays were performed on $7 \mathrm{H} 10$ medium $/ 1 \%$ glucose plates. Medium for the $m s h B$ mutant strain was supplemented with $25 \mu \mathrm{g}$ kanamycin $\mathrm{ml}^{-1}$ (Rawat et al., 2003). Liquid medium for the control strain, $\mathrm{Mc}^{2} 155$-gent, with a gentamicinresistance gene, and the mca mutant strain was supplemented with $10 \mu \mathrm{g}$ gentamicin $\mathrm{ml}^{-1}$, whilst solid medium was supplemented with $15 \mu \mathrm{g}$ gentamicin $\mathrm{ml}^{-1}$ (Rawat et al., 2004).

Tube and microdilution assays. Tube and microdilution assays were performed as described in the NCCLS guidelines (National Committee for Clinical Laboratory Standards, 1993).

Drug-toxicity assays. Drug toxicity was assessed by trypan blue exclusion staining of THP-1 monocytes and by fluorescence-activated cell sorting (FACS) analysis of propidium iodide (PI)-stained THP-1 cells exposed to increasing concentrations of the test drug. Growth of THP-1 cells, PI staining and FACS analysis were carried out as described previously (Pick et al., 2004).

THP-1 monocytes were grown as described previously (Pick et al., 2004). Different concentrations of drug were added to the medium and cells were exposed for $24 \mathrm{~h}$ at $37^{\circ} \mathrm{C}$. Cells were stained by adding
$0 \cdot 4 \%$ trypan blue and washed twice with PBS $(\mathrm{pH} 7 \cdot 4)$. Methanol was added to solubilize the stain and the absorbance of the methanol solution was measured at $605 \mathrm{~nm}$.

Mammalian cell-toxicity assays. Phorbol myristate acetate (PMA)-differentiated THP-1 macrophages were grown in modified RPMI in 24-well tissue culture plates to approximately $3 \times 10^{5}$ cells per well. Serial dilutions of EXEG1706 were added to test wells, whilst positive-control wells received $5 \mu \mathrm{g}$ cycloheximide and negativecontrol wells received $1 \%$ methanol. Plates were incubated for $24 \mathrm{~h}$ at $37^{\circ} \mathrm{C}$ in the presence of $5 \% \mathrm{CO}_{2}$. After incubation, the medium was removed from the wells and $150 \mu 10 \cdot 4 \%$ trypan blue in PBS was added to stain the remaining macrophages. Viability was determined by counting dead cells (cells retaining the stain) and live cells (unstained cells, which actively pump the stain out of the cell). The trypan blue was then removed, the wells were washed twice with PBS and $300 \mu \mathrm{l}$ methanol was added to each well. Aliquots $(200 \mu \mathrm{l})$ were transferred to an ELISA plate and the absorbance was measured at $605 \mathrm{~nm}$.

\section{RESULTS}

\section{Synthesis and antimicrobial activity of bromotyrosine alkaloids}

Thirty bromotyrosine alkaloid compounds were synthesized and evaluated for their ability to inhibit the growth of a number of micro-organisms (Arad \& Av-Gay, 2003). Five compounds (Fig. 1) were found to have good activity against the organisms tested, and two, EXEG1706 and EXEG 
Table 2. Diameters $(\mathrm{mm})$ of disk assay growth inhibition zones for various organisms after treatment with synthetic bromotyrosine alkaloids

Each disk contained $100 \mu \mathrm{g}$ test compound. ND, Not determined.

\begin{tabular}{|c|c|c|c|c|c|c|c|c|c|c|}
\hline Compound & \multicolumn{10}{|c|}{ Growth inhibition zone $(\mathrm{mm})$} \\
\hline EXEG1706 & 0 & 15 & 0 & 24 & 20 & 24 & 11 & 10 & 11 & 24 \\
\hline EXEG1707 & 0 & 20 & 7 & 19 & 17 & 19 & 9 & 9 & 10 & 16 \\
\hline EXEG1710 & 0 & 15 & 6 & 24 & 18 & 20 & 19 & 22 & 10 & ND \\
\hline
\end{tabular}

1710, were able to inhibit mycobacterial MCA in vitro (data not shown), confirming the results obtained by Nicholas et al. (2001).

Table 2 shows the results of disk sensitivity assays for these five compounds against several Gram-positive organisms, a Gram-negative organism and yeast. The best activity was observed against Gram-positive organisms, Staphylococci in particular. The compounds were generally less effective against Gram-negative organisms, including E. coli (Table 2), Enterococcus faecalis and Enterococcus faecium (see Table 4). One exception was EXEG3022, which had moderate activity against E. coli and was the only compound to have activity against the yeast, Saccharomyces cerevisiae. Of these five compounds of interest, EXEG1706 and EXEG1710 demonstrated the most potent activity against MRSA, meticillinsensitive $S$. aureus (MSSA) and vancomycin-resistant $S$. aureus (VRSA). EXEG1706 and EXEG1710 also had moderate activity against $M$. smegmatis and EXEG1706 was very effective against the mycobacterial vaccine strain, Mycobacterium bovis Bacille Calmette-Guérin (BCG; Table 2) and a clinical Streptococcus pneumoniae isolate (not shown in Table 2, $22 \mathrm{~mm}$ diameter zone of inhibition for $100 \mu \mathrm{g}$ compound applied to disk). Interestingly, EXEG1710 is a chlorotyrosine derivative with an otherwise identical chemical structure to EXEG1706.

The five bromotyrosine and chlorotyrosine alkaloids were tested further against MSSA, MRSA and VRSA. The MIC

Table 3. Growth inhibition by novel bromotyrosine antimicrobial compounds

\begin{tabular}{|c|c|c|c|}
\hline \multirow[t]{2}{*}{ Compound } & \multicolumn{3}{|c|}{$\operatorname{MIC}\left(\mu \mathrm{g} \mathrm{ml}^{-1}\right)$} \\
\hline & MSSA & MRSA & VRSA \\
\hline EXEG1706 & $2 \cdot 5-25$ & $2 \cdot 5-25$ & $2 \cdot 5-25$ \\
\hline EXEG1707 & 125 & 125 & 125 \\
\hline EXEG1710 & 100 & 100 & 100 \\
\hline EXEG1712 & 40 & 40 & 80 \\
\hline EXEG3022 & 50 & 50 & 70 \\
\hline
\end{tabular}

for each of the five alkaloids was obtained from a tube dilution assay carried out according to NCCLS guidelines (National Committee for Clinical Laboratory Standards, 1993). Table 3 shows that, while all compounds demonstrated effectiveness against these $S$. aureus strains, the compound with the lowest MICs was EXEG1706. Since EXEG1706 was the most promising antimicrobial from the group of novel bromotyrosine alkaloids tested, further studies were carried out with this compound.

\section{Growth inhibition of clinical pathogens by EXEG1706}

EXEG1706 antimicrobial activity was tested against an expanded panel of micro-organisms. Table 4 shows the MICs for EXEG1706 against a number of clinically important pathogens and M. smegmatis, which was grown in Middlebrook $7 \mathrm{H} 9$ medium rather than in BHI (see Methods). EXEG1706 MICs were between $2 \cdot 5$ and $25 \mu \mathrm{g} \mathrm{ml}{ }^{-1}$ for most organisms tested. As observed previously, this compound was not effective against the Gram-negative bacteria tested nor Enterococcus faecalis and Enterococcus faecium. It was

Table 4. Growth inhibition of expanded strain panel by EXEG1706

\begin{tabular}{|lc|}
\hline Micro-organism & MIC $\left(\boldsymbol{\mu g} \mathbf{~ m l}^{-\mathbf{1}}\right)$ \\
\hline Bacillus subtilis & Resistant \\
Enterococcus faecalis & Resistant \\
Enterococcus faecium & Resistant \\
Mycobacterium smegmatis MC $^{2} 155$ & $1 \cdot 5-15 \cdot 5$ \\
VRSA & $2 \cdot 5-25$ \\
MSSA & $8-16$ \\
MRSA, quinolone resistant & $4-16$ \\
MRSA, quinolone susceptible & $4-8$ \\
MRSA, heteroresistant & 8 \\
Staphylococcus epidermidis & $2 \cdot 5-25$ \\
VRSH & $2 \cdot 5-25$ \\
Streptococcus pneumoniae ATCC 49619 & Resistant \\
Streptococcus pneumoniae FC 579 & $2 \cdot 5-25$ \\
Staphylococcus saprophyticus & 2 \\
\hline
\end{tabular}


also ineffective against one strain of Streptococcus pneumoniae tested. Low MICs were observed for pathogenic $S$. aureus and for M. smegmatis (MIC of between 1.5 and $15 \cdot 5 \mu \mathrm{g} \mathrm{ml}^{-1}$ in Middlebrook 7H9 medium), encouraging further characterization of EXEG1706 antimicrobial activity.

\section{Sensitivity of staphylococci to EXEG1706}

Since most of the Staphylococcus spp. tested were quite sensitive to EXEG1706, the growth of three staphylococci was tested over a range of drug concentrations. Bacterial growth was monitored during a standard tube dilution assay by measuring the optical density of the culture at $605 \mathrm{~nm}$. Fig. 3 represents the growth of VRSA, Staphylococcus epidermidis and vancomycin-resistant Staphylococcus haemolyticus (VRSH) in the presence of EXEG1706 after $24 \mathrm{~h}$ at $37^{\circ} \mathrm{C}$. The growth of all three organisms was completely inhibited at $25 \mu \mathrm{g}$ EXEG1706 $\mathrm{ml}^{-1}$. For S. epidermidis, growth inhibition occurred down to a concentration of $0 \cdot 25 \mu \mathrm{g}$ EXEG1706 $\mathrm{ml}^{-1}$. For VRSH and VRSA, growth inhibition occurred down to the drug limits tested, i.e. $0 \cdot 025$ and $0 \cdot 0025 \mu \mathrm{g} \mathrm{ml}^{-1}$, respectively. This data indicated that VRSH and VRSA were the most sensitive to EXEG1706, as their growth was affected by very low concentrations of the drug.

\section{EXEG1706 activity against mycobacterial mutants in MSH-related genes}

Since the bromotyrosine compounds were based on compounds that inhibited MCA activity, we tested the activity of EXEG1706 on M. smegmatis strains in which we had inactivated the mca gene (Rawat et al., 2004) or interfered with MSH biosynthesis by inactivating the mca homologue, $m s h B$ (Rawat et al., 2003). In disk assays, EXEG1706 demonstrated significant activity against the wild-type strain at $50 \mu \mathrm{g} \mathrm{ml}^{-1}$, and higher activity against $m c a$ or $m s h B$ mutants, as seen in Table 5 .

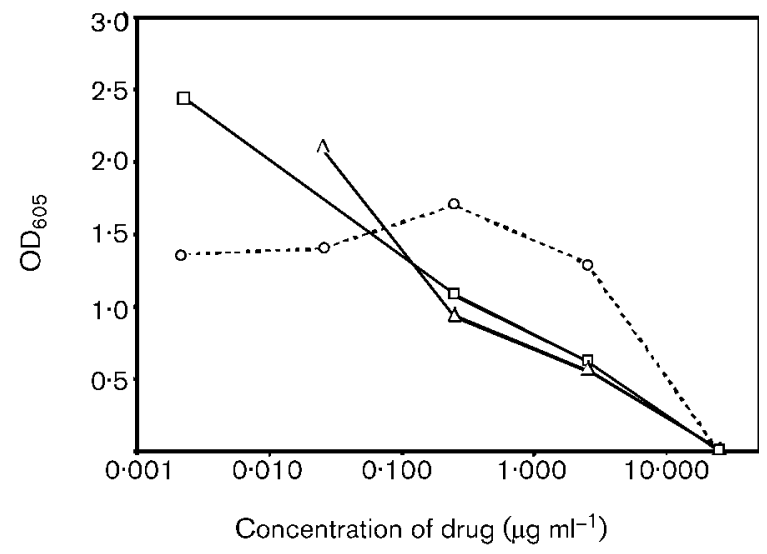

Fig. 3. Growth inhibition of staphylococci by EXEG1706. VRSA $(\square)$, VRSH $(\triangle)$ and $S$. epidermidis $(\bigcirc)$, were grown for $24 \mathrm{~h}$ in the presence of decreasing concentrations of EXEG1706. Bacterial growth was measured as $\mathrm{OD}_{605}$.

\section{EXEG1706 toxicity to macrophages and monocytes}

EXEG1706 toxicity to mammalian cells was determined by exposing adherent THP-1 human macrophages to $12 \cdot 5 \mu \mathrm{g}$ EXEG1706 $\mathrm{ml}^{-1}$. Compared with the solvent control, no decrease in adherent THP-1 cells was observed in the presence of EXEG1706 (data not shown), indicating that the drug was not toxic to the macrophages under these conditions.

This result was confirmed by the mammalian cell-toxicity assay, as described in Methods. Fig. 4(a) shows that below $100 \mu \mathrm{g}$ EXEG1706 $\mathrm{ml}^{-1}$, no significant macrophage toxicity was observed. Above $100 \mu \mathrm{g} \mathrm{ml}^{-1}, A_{605}$ increased with increasing drug concentration, indicating an increase in the number of dead macrophages stained with trypan blue. The insolubility of EXEG1706 in RPMI prevented testing of drug concentrations above $1000 \mu \mathrm{g} \mathrm{ml}^{-1}$. Under the conditions used, the $\mathrm{LD}_{50}$ of EXEG1706 was calculated to be $400 \mu \mathrm{g} \mathrm{ml}^{-1}$. EXEG1706 was more toxic to undifferentiated THP-1 monocytes when tested in the same manner, yielding an $\mathrm{LD}_{50}$ of $100 \mu \mathrm{g} \mathrm{ml}^{-1}$ (data not shown).

To determine the time required for EXEG1706 at its $\mathrm{LD}_{50}$ to have maximum toxic effect on PMA-differentiated THP-1 macrophages, the previous toxicity assay was carried out in the presence of $400 \mu \mathrm{g}$ EXEG1706 $\mathrm{ml}^{-1}$. Macrophage viability was assessed at 1, 2, 3, 4 and 24 h. As shown in Fig. 4(b), the maximum toxic effect of EXEG1706 at its $\mathrm{LD}_{50}$ occurred after $4 \mathrm{~h}$ of incubation.

EXEG1706 and the other novel bromotyrosine compounds were also tested for toxicity to THP-1 monocytes using a novel flow cytometry assay (Pick et al., 2004). Undifferentiated THP-1 monocytes were exposed to different concentrations of EXEG1706, EXEG1710, EXEG1712 or EXEG3022 at $37^{\circ} \mathrm{C}$ for $24 \mathrm{~h}$. The percentage of dead cells was monitored by PI staining and analysed by FACS, and the $\mathrm{LD}_{50}$ was calculated. EXEG1710 was least toxic with an $\mathrm{LD}_{50}$ of $300 \mu \mathrm{g} \mathrm{ml}^{-1}$. EXEG1706 was the next least toxic with an $\mathrm{LD}_{50}$ of $100 \mu \mathrm{g} \mathrm{ml}^{-1}$. Both EXEG1712 and EXEG3022 were more toxic to monocytes, with $\mathrm{LD}_{50}$ values of 40 and $70 \mu \mathrm{g} \mathrm{ml}^{-1}$, respectively. These two compounds had $\mathrm{LD}_{50}$

Table 5. Diameters $(\mathrm{mm})$ of disk assay growth inhibition zones for $M$. smegmatis parental wild-type strain, and $m s h B$ and mca mutant strains after treatment with EXEG1706

Data are presented as mean \pm range $(\mathrm{mm})$ of duplicate samples of exponential cells cultured in Middlebrook 7H9 medium. NA, Not available.

\begin{tabular}{|lrrcc|}
\hline Inhibitor $(\boldsymbol{\mu g})$ & $\mathbf{M c}^{2} \mathbf{1 5 5}$ & $\boldsymbol{m} \boldsymbol{s h} \boldsymbol{B}$ & $\mathbf{M c}^{\mathbf{2}}$ 155-gent & $\boldsymbol{m c a}$ \\
\hline $10 \cdot 0$ & $5 \cdot 9 \pm 0 \cdot 0$ & $7 \cdot 3 \pm 1 \cdot 2$ & $21 \cdot 0 \pm 1 \cdot 0$ & $28 \cdot 0 \pm 1 \cdot 5$ \\
$50 \cdot 0$ & $15 \cdot 0 \pm 0 \cdot 0$ & $20 \cdot 0 \pm 0 \cdot 0$ & $31 \cdot 5 \pm 0 \cdot 5$ & $37 \cdot 3 \pm 1 \cdot 8$ \\
$100 \cdot 0$ & $18 \cdot 5 \pm 0.5$ & $26 \cdot 5 \pm 0 \cdot 5$ & $\mathrm{NA}$ & $\mathrm{NA}$ \\
\hline
\end{tabular}



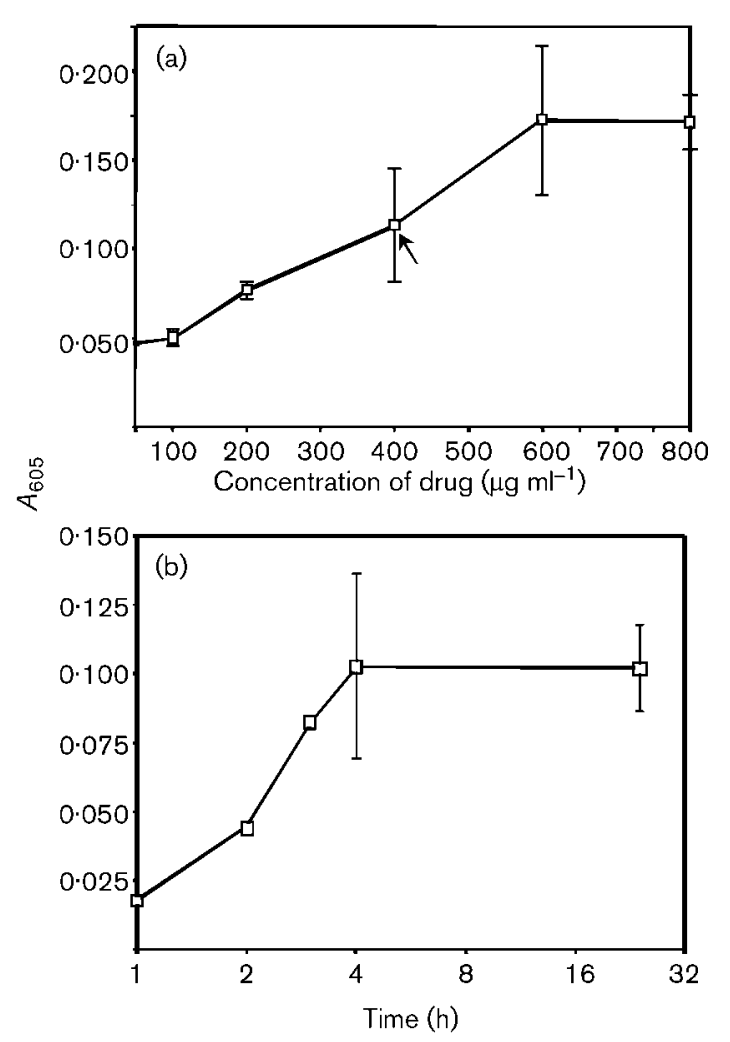

Fig. 4. EXEG1706 toxicity for differentiated THP-1 macrophages. (a) THP-1 macrophages were exposed to increasing concentrations of EXEG1706 for $24 \mathrm{~h}$, stained with trypan blue and washed. The arrow denotes the $\mathrm{LD}_{50}$ value. (b) Time taken to achieve maximum toxicity for EXEG1706 at the $L_{50}$. THP-1 macrophages were exposed to EXEG1706 at its $L_{50}$ $\left(400 \mu \mathrm{g} \mathrm{ml}^{-1}\right)$. Samples were taken at various intervals, stained with trypan blue and washed. The number of dead (stained) cells was measured by determining $A_{605}$. Results are shown as the mean \pm SEM for triplicate measurements.

values very close to their MIC values (Table 3 ), whereas EXEG1706 and EXEG1710 had MIC values considerably below their $\mathrm{LD}_{50}$ values. As EXEG1706 had the lowest MIC values, it was considered the most promising antimicrobial drug from this group.

\section{DISCUSSION}

Bacterial resistance to antibiotics is a serious public health concern because of nosocomial pathogens and organisms infecting those with immune systems weakened by disease or genetic disposition. M. tuberculosis causes significant disease among the AIDS and homeless populations. The worldwide incidence of TB is 8 million people a year, its prevalence is 1.86 billion people and the case fatality rate is $23 \%$ (Dye et al., 1999). Because of side effects and the length of time required for current TB treatment, patient noncompliance is common and contributes to the spread of multi-drug-resistant TB. Similarly, drug-resistant $S$. aureus strains are a major health concern. $S$. aureus is a problematic nosocomial pathogen and this is complicated further by the fact that humans are a natural reservoir for this organism (Lowy, 1998). Both MSSA and MRSA are persistent colonizers that can be carried asymptomatically, increasing the risk of subsequent infection for those colonized (Casewell \& Hill, 1986; Sanford et al., 1994; Wenzel \& Perl, 1995). As with M. tuberculosis, individuals colonized with $S$. aureus often have immune deficiencies or other complicating health factors such as type 1 diabetes, intravenous drug usage, surgical interventions, haemodialysis and AIDS (Kluytmans et al., 1995; Tuazon \& Sheagren, 1974; Tuazon et al., 1975; Weinke et al., 1992; Yu et al., 1986). Moreover, the emergence of VRSA is a near reality, as $S$. aureus isolates with intermediate sensitivity to vancomycin (Bartley, 2002; Mallaval et al., 2004) and vancomycinresistant coagulase-negative staphylococci have already been reported (Schwalbe et al., 1987).

For the reasons outlined above, it has become even more important to identify new bacterial drug targets and develop new antimicrobial agents. A bromotyrosine alkaloid found in an extract from a non-verongid sponge inhibited the activity of mycobacterial MCA (Nicholas et al., 2001). A series of synthetic bromotyrosine alkaloids was synthesized, modelled on the compound from the sponge extract, and their antimicrobial activities were evaluated (Arad \& AvGay, 2003; Kende et al., 2004). Five compounds from this series (Table 1) had good activity against a range of Grampositive organisms, as demonstrated in disk diffusion assays (Table 2). None of the compounds was particularly effective against the Gram-negative organisms tested, suggesting either that their molecular target is absent in Gram-negative bacteria or that the compounds lack the ability to cross the Gram-negative cell wall.

M. smegmatis mutants defective in MSH biosynthesis have increased sensitivity to xenobiotics and alkylating agents (Rawat et al., 2002). In our studies, a mutant disrupted in $m s h B$, a gene paralogous to $m c a$ and involved in MSH biosynthesis, demonstrated increased sensitivity to EXEG1706 compared with the control, suggesting that MSH and MCA are involved in the protection against the bromotyrosine compounds. Furthermore, a mutant disrupted in $m c a$ also showed increased sensitivity. This suggests that the bromotyrosine compounds act on another, as yet unidentified, cellular target in mycobacteria and are not specific inhibitors of MCA. Since staphylococci do not produce MSH (Newton et al., 1996) and there is no complete S. aureus MCA homologue, it is possible that another enzyme or set of enzymes serves as the target for these broad-range antimicrobial agents.

We took a bioinformatics approach to identify possible drug targets for the bromotyrosine compounds in Grampositive bacteria. The identified in vitro target (MCA) and the MSH biosynthesis enzyme MshB have identical active sites situated around a zinc ion (Maynes et al., 2003). Scrutiny of the MshB crystal structure has revealed a large family of related zinc hydrolases with a novel fold existing 
among the Gram-positive bacteria (Maynes et al., 2003; McCarthy et al., 2003). A BLAST search using M. tuberculosis MCA revealed several homologues within staphylococci containing two active site motifs, HPDDE and HPDH (Maynes et al., 2003), essential for the amide hydrolase reaction (data not shown). Since the synthetic bromotyrosine compound EXEG1706 is centred on an oxime moiety (hydroxamate) that binds metals, it is likely to be able to inhibit zinc metalloenzymes in other Gram-positive bacteria structurally related to $M$. tuberculosis MCA.

Interestingly, EXEG1706 and EXEG1710 gave rise to some of the largest zones of inhibition for the staphylococci and mycobacteria tested. These two compounds have an identical structure except for the presence of two bromines (EXEG1706) or chlorines (EXEG1710) substituted on the tyrosine moiety of each molecule. This suggests that this alkaloid structure is exceptionally active against these organisms. When MICs were calculated for each compound against MSSA, MRSA and VRSA, EXEG1706, EXEG1712 and EXEG3022 had the lowest MICs (Table 3). EXEG1706 demonstrated the lowest MICs of all, $2 \cdot 5 \mu \mathrm{g} \mathrm{ml}^{-1}$ for all three organisms, making it the most potent of the five compounds, and thus became the lead antimicrobial compound and was characterized further for range of effectiveness and mammalian-cell toxicity.

EXEG1706 MICs were determined by standard tube assay (National Committee for Clinical Laboratory Standards, 1993). The results in Table 4 confirmed that EXEG1706 did not appear to be effective against Gram-negative organisms. It was also ineffective against Bacillus subtilis and one strain of Streptococcus pneumoniae (ATCC 49619); however, against all other organisms tested, EXEG1706 had a MIC of less than $25 \mu \mathrm{g} \mathrm{ml}^{-1}$. This group included M. smegmatis, MSSA, quinolone-susceptible and -resistant MRSA, heteroresistant MRSA, VRSA and VRSH. In addition to the effectiveness of EXEG1706 against mycobacteria, its effectiveness against multi-drug-resistant staphylococci is of great interest. The low MIC of EXEG1706 against these organisms means that the dose of drug used in humans potentially could be kept low, minimizing mammalian-cell toxicity complications. To test the ability of EXEG1706 to inhibit growth, VRSA, VRSH and S. epidermidis were grown in the presence of decreasing concentrations of this bromotyrosine alkaloid. Only $25 \mu \mathrm{g}$ EXEG1706 $\mathrm{ml}^{-1}$ completely inhibited growth of all three organisms (Fig. 3). The growth of $S$. epidermidis was inhibited down to a concentration of $0 \cdot 25 \mu \mathrm{g}$ EXEG1706 $\mathrm{ml}^{-1}$, whereas the growth of VRSA and VRSH was inhibited down to 0.0025 and $0.025 \mu \mathrm{g} \mathrm{ml}^{-1}$, respectively. This indicated that VRSA and VRSH were the most sensitive to this compound.

The sensitivity of mammalian cells to EXEG1706 was determined by exposing PMA-differentiated THP-1 macrophages to increasing concentrations of the compound (Fig. 4a). Toxicity was observed only above $100 \mu \mathrm{g}$ EXEG1706 $\mathrm{ml}^{-1}$ and the $\mathrm{LD}_{50}$ was calculated to be $400 \mu \mathrm{g} \mathrm{ml}^{-1}$. This $\mathrm{LD}_{50}$ value is at least 16 -fold greater than effective concentrations of EXEG1706, as demonstrated in growth-inhibition assays with VRSA, VRSH and S. epidermidis and compared with the calculated MICs for staphylococci and mycobacteria from standard tube dilution assays. In addition, adherent THP-1 macrophages were not affected by $12.5 \mu \mathrm{g}$ EXEG1706 $\mathrm{ml}^{-1}$ (data not shown). After $4 \mathrm{~h}$ in the presence of EXEG1706 at its LD $_{50}$, THP-1 macrophages exhibited maximum toxicity to this drug (Fig. 4b). Flow cytometry data demonstrating the toxicity of EXEG1706, EXEG1710, EXEG1712 and EXEG3022 on undifferentiated THP-1 monocytes has been reported previously (Pick et al., 2004). With the exception of EXEG1710, EXEG1706 was the least toxic compound. For unknown reasons, EXEG1706 was also approximately four times more toxic to THP-1 monocytes than to macrophages, with an $\mathrm{LD}_{50}$ of $100 \mu \mathrm{g} \mathrm{ml}^{-1}$ for THP-1 monocytes. In the process of differentiating into macrophages, monocytes undergo changes in cell-surface marker expression, and macrophages have elevated endocytic and phagocytic capacity (Basta et al., 1999; Coccia et al., 1999; Gessani et al., 1993). These changes may contribute to cell-specific differences in EXEG1706 sensitivity due to drug uptake, drug stability in the cells or availability of a toxicity target. That the EXEG1706 MICs are considerably below its $\mathrm{LD}_{50}$ indicates that this compound potentially could be used against antibiotic-resistant staphylococci and mycobacteria.

In this study, we have reported the synthesis and characterization of a series of bromotyrosine alkaloids based on a naturally occurring alkaloid. This novel class of antimicrobials shows promise against Gram-positive organisms, including mycobacteria and staphylococci, including MRSA, VRSA and VRSH. EXEG1706 emerged as the lead compound due to its low MICs against almost all Gram-positive organisms tested and its low toxicity for THP-1 cells. The lack of options available to treat infections caused by antibioticresistant, Gram-positive organisms makes it important to identify new classes of antimicrobials. EXEG1706 was crystallized in its trifluoroacetate salt and obviously a less dangerous salt form of this family of compounds needs to be designed. Nevertheless, the bromotyrosine alkaloid class, as demonstrated by the lead compound EXEG1706, was identified in vitro as an antimicrobial that provides new options for the treatment of multi-drug-resistant, Grampositive infections. Animal studies are the next step in the evaluation of this family of compounds.

\section{ACKNOWLEDGEMENTS}

The authors would like to thank Dr Barb Conway for data analysis and assistance with manuscript preparation. This work was supported by a grant to Y.A.-G. from eXegenics Pharmaceuticals Inc. and the TB Veterans Charitable Foundation. Y.A.-G. is a Canadian Institute of Health Research and British Columbia Lung Association Scholar.

\section{REFERENCES}

Arad, D. \& Av-Gay, Y. (2003). Antimicrobial compounds against Staphylococci, Mycobacteria and other infectious agents. US patent application WO 2004012506 and WO 2003087127. 
Bartley, J. (2002). First case of VRSA identified in Michigan. Infect Control Hosp Epidemiol 23, 480.

Basta, S., Knoetig, S. M., Spagnuolo-Weaver, M., Allan, G. \& McCullough, K. C. (1999). Modulation of monocytic cell activity and virus susceptibility during differentiation into macrophages. J Immunol 162, 3961-3969.

Casewell, M. W. \& Hill, R. L. (1986). The carrier state: methicillinresistant Staphylococcus aureus. J Antimicrob Chemother 18 (Suppl. A), 1-12.

Cassone, M., Campanile, F., Pantosti, A., Venditti, M. \& Stefani, S. (2004). Identification of a variant "Rome clone" of methicillinresistant Staphylococcus aureus with decreased susceptibility to vancomycin, responsible for an outbreak in an intensive care unit. Microb Drug Resist 10, 43-49.

Coccia, E. M., Del Russo, N., Stellacci, E., Testa, U., Marziali, G. \& Battistini, A. (1999). STAT1 activation during monocyte to macrophage maturation: role of adhesion molecules. Int Immunol 11, 1075-1083.

Dye, C., Scheele, S., Dolin, P., Pathania, V. \& Raviglione, M. C. (1999). Consensus statement. Global burden of tuberculosis: estimated incidence, prevalence, and mortality by country. WHO Global Surveillance and Monitoring Project. JAMA 282, 677-686.

Gessani, S., Testa, U., Varano, B. \& 7 other authors (1993). Enhanced production of LPS-induced cytokines during differentiation of human monocytes to macrophages. Role of LPS receptors. J Immunol 151, 3758-3766.

Kende, A. S., Lan, J. \& Fan, J. (2004). Total synthesis of a dibromotyrosine alkaloid inhibitor of mycothiol S-conjugate amidase. Tetrahedron Lett 45, 133-135.

Kluytmans, J. A., Mouton, J. W., ljzerman, E. P., VandenbrouckeGrauls, C. M., Maat, A. W., Wagenvoort, J. H. \& Verbrugh, H. A. (1995). Nasal carriage of Staphylococcus aureus as a major risk factor for wound infections after cardiac surgery. J Infect Dis 171, 216-219.

Lowy, F. D. (1998). Staphylococcus aureus infections. N Engl J Med 339, 520-532.

Mallaval, F. O., Carricajo, A., Delavenna, F. \& 7 other authors (2004). Detection of an outbreak of methicillin-resistant Staphylococcus aureus with reduced susceptibility to glycopeptides in a French hospital. Clin Microbiol Infect 10, 459-461.

Maynes, J. T., Garen, C., Cherney, M. M., Newton, G., Arad, D., Av-Gay, Y., Fahey, R. C. \& James, M. N. (2003). The crystal structure of 1-D-myo-inosityl 2-acetamido-2-deoxy- $\alpha$-D-glucopyranoside deacetylase (MshB) from Mycobacterium tuberculosis reveals a zinc hydrolase with a lactate dehydrogenase fold. J Biol Chem 278, 47166-47170.

McCarthy, A. A., Knijff, R., Peterson, N. A. \& Baker, E. N. (2003). Crystallization and preliminary $\mathrm{X}$-ray analysis of $\mathrm{N}$-acetyl-1-D-myoinosityl-2-deoxy- $\alpha$-D-glucopyranoside deacetylase (MshB) from Mycobacterium tuberculosis. Acta Crystallogr D Biol Crystallogr 59, 2316-2318.

National Committee for Clinical Laboratory Standards (1993). Performance standards for antimicrobial susceptibility tests for bacteria that grow aerobically, approved standard M7-T2, 2nd edn. Villanova, PA: National Committee for Clinical Laboratory Standards.

Negi, S. S., Gupta, S. \& Lal, S. (2003). Drug resistance in tuberculosis in Delhi: a 2 year profile (2001-2002). J Commun Dis 35, 74-81.

Newton, G. L., Arnold, K., Price, M. S. \& 7 other authors (1996). Distribution of thiols in microorganisms: mycothiol is a major thiol in most actinomycetes. J Bacteriol 178, 1990-1995.
Newton, G. L., Av-Gay, Y. \& Fahey, R. C. (2000). A novel mycothioldependent detoxification pathway in mycobacteria involving mycothiol S-conjugate amidase. Biochemistry 39, 10739-10746.

Nicholas, G. M., Newton, G. L., Fahey, R. C. \& Bewley, C. A. (2001). Novel bromotyrosine alkaloids: inhibitors of mycothiol $S$-conjugate amidase. Org Lett 3, 1543-1545.

Nicholas, G. M., Eckman, L. L., Ray, S., Hughes, R. O., Pfefferkorn, J. A., Barluenga, S., Nicolaou, K. C. \& Bewley, C. A. (2002). Bromotyrosine-derived natural and synthetic products as inhibitors of mycothiol-S-conjugate amidase. Bioorg Med Chem Lett 12, 2487-2490.

Pick, N., Cameron, S., Arad, D. \& Av-Gay, Y. (2004). Screening of compounds toxicity against human monocytic cell line-THP-1 by flow cytometry. Biol Proced Online 6, 220-225.

Rawat, M., Newton, G., Ko, M., Martinez, G., Fahey, R. C. \& Av-Gay, Y. (2002). Mycothiol-deficient Mycobacterium smegmatis mutants are hypersensitive to alkylating agents, free radicals, and antibiotics. Antimicrob Agents Chemother 46, 3348-3355.

Rawat, M., Kovacevic, S., Billman-Jacobe, H. \& Av-Gay, Y. (2003). Inactivation of $m s h B$, a key gene in the mycothiol biosynthesis pathway in Mycobacterium smegmatis. Microbiology 149, 1341-1349.

Rawat, M., Uppal, M., Newton, G., Steffek, M., Fahey, R. C. \& Av-Gay, Y. (2004). Targeted mutagenesis of the Mycobacterium smegmatis mca gene, encoding a mycothiol-dependent detoxification protein. J Bacteriol 186, 6050-6058.

Sanford, M. D., Widmer, A. F., Bale, M. J., Jones, R. N. \& Wenzel, R. P. (1994). Efficient detection and long-term persistence of the carriage of methicillin-resistant Staphylococcus aureus. Clin Infect Dis 19, 1123-1128.

Schwalbe, R. S., Stapleton, J. T. \& Gilligan, P. H. (1987). Emergence of vancomycin resistance in coagulase-negative staphylococci. $N$ Engl J Med 316, 927-931.

Tuazon, C. U. \& Sheagren, J. N. (1974). Increased rate of carriage of Staphylococcus aureus among narcotic addicts. J Infect Dis 129, 725-727.

Tuazon, C. U., Perez, A., Kishaba, T. \& Sheagren, J. N. (1975). Staphylococcus aureus among insulin-injecting diabetic patients. An increased carrier rate. JAMA 231, 1272.

Wagner, J., Kallen, J., Ehrhardt, C., Evenou, J. P. \& Wagner, D. (1998). Rational design, synthesis, and X-ray structure of selective noncovalent thrombin inhibitors. J Med Chem 41, 3664-3674.

Weinke, T., Schiller, R., Fehrenbach, F. J. \& Pohle, H. D. (1992). Association between Staphylococcus aureus nasopharyngeal colonization and septicemia in patients infected with the human immunodeficiency virus. Eur J Clin Microbiol Infect Dis 11, 985-989.

Wenzel, R. P. \& Perl, T. M. (1995). The significance of nasal carriage of Staphylococcus aureus and the incidence of postoperative wound infection. J Hosp Infect 31, 13-24.

World Health Organization (2002). Tuberculosis, WHO fact sheet no. 104. Geneva: World Health Organization.

Yanisch-Perron, C., Vieira, J. \& Messing, J. (1985). Improved M13 phage cloning vectors and host strains: nucleotide sequences of the M13mp18 and pUC19 vectors. Gene 33, 103-119.

Yu, V. L., Goetz, A., Wagener, M., Smith, P. B., Rihs, J. D., Hanchett, J. \& Zuravleff, J. J. (1986). Staphylococcus aureus nasal carriage and infection in patients on hemodialysis. Efficacy of antibiotic prophylaxis. N Engl J Med 315, 91-96. 